\title{
CONDIÇÕES DO TRABALHO, SAÚDE E BEM ESTAR DOS TRABALHADORES DE ENFERMAGEM NA EUROPA (NURSES'EARLY EXIT STUDY)
}

\author{
Donatella Camerino $^{1}$, Leila Maria Mansano Sarquis², Giovanni Costa ${ }^{3}$
}

RESUMO: A escassez de trabalhadores da enfermagem e um elevado turnover despertaram preocupação na Europa. O Nurses'Early Exit Study-NEXT. Estudo multicêntrico, intercultural e longitudinal. Participaram 10 países da Europa. O objetivo foi observar a relação entre condições de trabalho e saúde dos trabalhadores de enfermagem. Os dados de 19.099 trabalhadores de enfermagem foram analisados por meio da análise descritiva e da regressão linear. Os resultados demonstraram que a saúde e o bem estar dos trabalhadores de enfermagem dependem das condições do trabalho. A fim de planejar as intervenções que podem reduzir o turnover e a deficiência de trabalhadores de enfermagem na Europa. É importante que se considere o processo das atividades laborativas para o desenvolvimento do trabalho até a aposentadoria, situação que pode reduzir o abandono precoce no trabalho, bem como reduzir a carência significativa destes trabalhadores existente nesta área.

PALAVRAS-CHAVE: Saúde do trabalhador; Enfermagem; Condições de trabalho.

\section{NURSES' WORKING CONDITIONS, HEALTH AND WELL BEING IN EUROPE (NURSES'EARLY EXIT STUDY)}

\begin{abstract}
The shortage of workers in nursing and a high turnover aroused concern in Europe. The Nurses'Early Exit Study (NEXT). Multicenter, intercultural and longitudinal study. Ten European countries participated. The objective was to observe the relationship between working conditions and nursing workers' health. Data from 19.099 nursing workers were analyzed by descriptive analysis and linear regression. The results showed that health and well being of nursing workers depends on conditions of work. In order to plan interventions that can reduce the "turnover" and disability among nursing workers in Europe. It is important to consider the process of labor activities for the development of work until retirement, which may reduce the early work exit, as well as reduce the significant shortage of workers in this area. KEYWORDS: Occupational health; Nursing; Working conditions.

\section{CONDICIONES DE TRABAJO, SALUD Y EL BIENESTAR DE LOS TRABAJADORES DE ENFERMERÍA EN EUROPA (NURSES'EARLY EXIT STUDY)}

RESUMEN: La escasez de trabajadores de enfermería y el elevado volumen de facturación despertó preocupación en Europa. El Nurses'Early Exit Study-NEXT, estudio multicéntrico, intercultural y longitudinal. Participaron 10 países europeos. El objetivo fue observar la relación entre las condiciones de trabajo y la salud de los trabajadores de enfermería. Los datos de 19.099 trabajadores de enfermería fueron analizados por medio del análisis descriptivo y de la regresión lineal. Los resultados mostraron que la salud y el bienestar de los trabajadores de enfermería dependen de las condiciones de trabajo. Con el fin de planificar las intervenciones que pueden reducir el volumen de negocios y la deficiencia de trabajadores de enfermería en Europa. Es importante considerar el proceso de las actividades laborales para el desarrollo del trabajo hasta la jubilación, situación que puede reducir el abandono precoz en el trabajo, así como reducir la gran escasez de trabajadores en este sector.

PALABRAS CLAVE: Salud del trabajador, Enfermería, Condiciones de trabajo.

${ }^{1}$ Psicóloga. Professora do Departamento de Medicina do Trabalho da Università degli Studi di Milano-UNIMI e do Ospedale Maggiore, Policlinico Mangiagalli e Regina Elena.

${ }^{2}$ Doutora em Enfermagem. Professor Adjunto da Universidade Federal do Paraná-UFPR. Membro do Grupo de Estudo Multidisciplinar de Saúde do Adulto-GEMSA do Grupo de Pesquisa de Qualidade de Vida do Departamento da Escola de Enfermagem. Universidade de São Paulo-USP.

${ }^{3}$ Médico do Trabalho. Professor do Departamento de Medicina do Trabalho da Università degli Studi di Milano- UNIMI e do Ospedale Maggiore, Policlinico Mangiagalli e Regina Elena.

Autor correspondente:

Donatella Camerino

Via San Barnaba, 8 - 20-122 - Milano-Italia

Recebido: 21/07/08

E-mail: donatella.camerino@unimi.it

Aprovado: 20/03/09 


\section{INTRODUÇÃO}

Na Europa, nos primeiros anos do século XXI existia uma grande carência de trabalhadores de enfermagem com número reduzido entre os jovens para a escolha profissional, bem como o abandono precoce dos trabalhadores de enfermagem que iniciavam a atividade laborativa em instituições de saúde.

A Organização Internacional para a Cooperação Economia e Desenvolvimento(1) considerou que as principais razões para esta situação estavam relacionadas com a globalização, com o crescimento, com o crescente envelhecimento da população, com o progresso tecnológico e a crescente expectativa de vida da população.

Entre 2002 e 2004, o estudo denominado Nurses'Early Exit Study (NEXT) teve como objetivo caracterizar as razões, circunstâncias e conseqüências do abandono dos trabalhadores de enfermagem na profissão. O estudo teve financiamento inicial pelo Joint Programme for Working Life Research in Europe (SALTSA) e sucessivamente da União Européia (QLK6-CT-2001-00475), considerada a grave carência dos trabalhadores de enfermagem e da necessidade de encontrar soluções para o futuro desta profissão na Europa ${ }^{(2)}$.

O projeto foi coordenado pela Universidade de Wuppertal (Alemanha). Foi realizado simultaneamente na Bélgica (BE), Finlandia (FIN), França (FR), Alemanha (DE), Grã Bretanha (UK), Itália (IT), Países Baixos (NL), Polônia (POL), Suécia (SW) e Eslováquia (SLK). A Noruega $(\mathrm{N})$ colaborou na qualidade de membro associado. A porcentagem de trabalhadores de enfermagem que manifestaram intenção de deixar a profissão variou de país para país, a porcentagem mais alta ocorreu na Itália (20,7\%); a mais baixa na Holanda $(8,8 \%)$ e na Bélgica (9,8\%); este fato se dá provavelmente porque nestes dois países, os trabalhadores de enfermagem podem desenvolver suas atividades laborais em expediente laboral reduzido e com horários mais flexíveis. As razões relatadas pelos trabalhadores de enfermagem que abandonavam a profissão estavam relacionadas, sobretudo às condições do trabalho e problemas econômicos ${ }^{(3)}$.

Para a maioria dos países considerados, os trabalhadores de enfermagem acima de 50 anos, tinham expressado a intenção de buscar, por conta própria, alternativas para melhorar de vida ou trabalho, por causa das condições desfavoráveis no trabalho (em particular a sobrecarga de trabalho) e/ou por causa das más condições de saúde (apesar das leis européias protegerem o trabalhador com agravos à saúde). Vários jovens expressaram a necessidade de mudança de emprego a fim de enriquecer seu perfil profissional ou para melhorar a própria vida pessoal e familiar ${ }^{(4)}$.

O turnover apresentado entre os trabalhadores de enfermagem caracteriza-se pela alternação da equipe de funcionários em um local de trabalho determinado.É um conceito mais amplo do que a "abandono na profissão”. Um trabalhador de enfermagem pode deixar definitivamente a profissão pelo abandono do trabalho, mudança do tipo de atividade ou pela aposentadoria, mas também pode decidir continuar na profissão e mudar somente o local de trabalho dentro da própria instituição ou em outra agência, o que não acontece na Europa.

Deixar um posto de trabalho pode ser uma escolha voluntária se o trabalhador de enfermagem deseja melhorar suas condições de trabalho e de salário para seguir a família ou se transferir para outra localidade ${ }^{(5)}$, mas igualmente pode ser uma imposição devido à reorganização profissional, insatisfação no trabalho, e um estado de incapacidade em administrar outras situações do cotidiano oriundas da atividade laborativa. Na literatura, o descontentamento na atmosfera de trabalho é a razão mais importante do abandono, em detrimento das razões demográficas ou pessoais ${ }^{(3,6-7)}$.

Geralmente, o alto turnover determina um agravamento da qualidade do serviço assistencial, causando dano à imagem da companhia e à saúde dos trabalhadores. Envolve problemas de organização, custos diretos e indiretos ${ }^{(8)}$. Os trabalhadores de enfermagem que permanecem são forçados a aumentar o ritmo de trabalho a fim de manter os níveis precedentes de produtividade, a adaptar-se aos horários laborais prolongados ou a uma troca de turnos não adequados às próprias necessidades de recuperação e, eventualmente, devem ficar encarregados também com a formação e sustentação de novos trabalhadores ${ }^{(6)}$. A substituição de trabalhadores pode interferir negativamente com o estabelecimento de relações sociais contínuas nos grupos de trabalho. A perda de coesão na equipe e familiaridade entre seus membros pode conduzir a episódios de intolerância e agressividade contra supervisores, subordinados e colegas; este agravamento no clima organizacional pode transformar-se em causa posterior para futuro abandono na profissão ${ }^{(9)}$.

A situação gerada por alto turnouver pode, conseqüentemente, ter repercussões negativas também na saúde da equipe de trabalhadores. Em particular, uma carga excessiva de trabalho físico e 
mental envolve-os de tensão e, em longo prazo, causa efeitos graves como doenças cardiovasculares, osteoarticulares, gastrointestinais, depressão, ansiedade, e Síndrome de Burnout ${ }^{(10)}$.

Carga de trabalho elevada e contínuo estresse são também fatores desencadeadores de exposição ocupacional $^{(11-12)}$ e podem desencadear situações geradoras de conflitos entre pacientes e familiares quando o atendimento realizado não é qualificado ${ }^{(13-14)}$.

O objetivo do estudo foi verificar as condições de saúde e de bem estar dos trabalhadores de enfermagem na Europa (população NEXT) em relação às condições de trabalho, a fim de compreender as razões do elevado turnover observado nesta população.

\section{METODOLOGIA}

A maioria dos países que participaram do estudo NEXT aderiu ao seu desenho intercultural, longitudinal e aplicaram a mesma metodologia do estudo.

A seleção foi realizada de maneira que, nos vários países, fosse proporcionada distribuição integral da população de trabalhadores de enfermagem, assim como a distribuição uniforme entre os vários tipos das instituições sanitárias e entre as várias áreas territoriais. Participaram do estudo 147 hospitais, 185 asilos, 76 instituições de atendimento domiciliar, 177 ambulatórios, totalizando 585 instituições entrevistadas.

Foram envolvidos 30.497 trabalhadores de enfermagem de instituições hospitalares; 3.958 de instituições asilares e 5.281 empregados nas instituições territoriais caracterizados em ambulatórios e prestadores de assistência domiciliar. Deste estudo foram removidos os dados vindos da Grã Bretanha, Finlândia e Noruega, pois estes não alcançaram uma resposta suficiente e não atenderam ao protocolo pré-determinado pelo estudo.

$\mathrm{O}$ instrumento final utilizado para a coleta de dados foi elaborado a partir de um estudo piloto municipal preliminar realizado na França, na Itália e na Alemanha, em 2001. As perguntas do questionário relativas às condições do trabalho pertencem principalmente ao questionário psicossocial Copenhagen $^{(15)}$ que investiga condições de trabalho, realização profissional, relações sociais, carência emocional, plenitude e significado das tarefas, grau de autonomia no trabalho, qualidade da liderança, satisfação de trabalho, conflitos do cargo, sobrecarga de trabalho, grau de participação e interesse pela profissão e pela própria organização do trabalho.

A fim de avaliar conflito entre a casa/trabalho e trabalho/casa foi utilizada a escala Work Family Conflict $^{(16)}$. As perguntas específicas relacionadas ao trabalho foram esboçadas, parcialmente, do questionário psicossocial de Copenhagen ${ }^{(15)}$ e parcialmente formuladas para o grupo de estudo NEXT. A estes, foram adicionadas perguntas referentes à autonomia, conflito entre pacientes e trabalhadores e a rotatividade de turnos no trabalho.

As informações sobre os recursos individuais e o estado de saúde e bem estar foram embasadas no referencial de Effort-Reward-Imbalance ${ }^{(17)}$ para a variável "insatisfação no trabalho, reconhecimento no trabalho e incapacidade do sujeito se desligar das preocupações do trabalho" foi utilizada a escala Copenhagen Burnout Inventory, General Health e Work Ability Index ${ }^{(18)}$ para analisar "o índice de capacidade funcional e a exaustão emocional”. A análise da variável “saúde em geral”, foi mensurada pelo General Health of Copenhagen Psychosocial Questionnaire(15) (Tabela 1).

Tabela 1 - Escalas empregadas para a avaliação do estado de saúde e do bem estar dos trabalhadores de enfermagem. NEXT, 2005

\begin{tabular}{|c|c|c|}
\hline Medida & Escala & Pontuação \\
\hline $\begin{array}{l}\text { Exaustão } \\
\text { emocional }\end{array}$ & $\begin{array}{l}\text { Copenhagen } \\
\text { Burnout } \\
\text { Inventory(20) }\end{array}$ & $\begin{array}{l}\text { Varia de } 1-5 \text {, uma } \\
\text { contagem mais } \\
\text { elevada indica maior } \\
\text { exaustão psico-física }\end{array}$ \\
\hline $\begin{array}{l}\text { Saúde em } \\
\text { geral }\end{array}$ & $\begin{array}{l}\text { General Health of } \\
\text { Copenhagen } \\
\text { Psychosocial } \\
\text { Questionnaire }^{(15)}\end{array}$ & $\begin{array}{l}\text { Varia de } 0-100 \text {, uma } \\
\text { contagem mais alta } \\
\text { indica um melhor } \\
\text { estado de saúde. }\end{array}$ \\
\hline $\begin{array}{l}\text { Índice de } \\
\text { capacidade } \\
\text { funcional }\end{array}$ & $\begin{array}{l}\text { Work Ability } \\
\text { Index }^{(18)} \text { versão } \\
\text { reduzida }^{(19)}\end{array}$ & $\begin{array}{l}07-27=\text { decadente } \\
28-36=\text { moderado } \\
37-43=\text { bom } \\
44-49=\text { excelente }\end{array}$ \\
\hline
\end{tabular}

O estudo teve a aprovação do Comitê de Ética da Universidade de Wuppertal (Alemanha). A participação dos trabalhadores de enfermagem foi voluntária e a aplicação e o recolhimento dos questionários foram conduzidos respeitando o sigilo das informações e do anonimato. O instrumento foi coletado em duas etapas, sendo reaplicado após um ano. Cada trabalhador de enfermagem obteve um código; construído com base nos nomes e datas de nascimento do seu país.

Após a primeira avaliação em 2002/2003, 4.227 enfermeiras saíram do local de trabalho. O 
questionário de seguimento foi emitido aos 59.510 trabalhadores de enfermagem restantes. Os trabalhadores foram monitorados e, após um ano, suas condições de saúde e bem-estar foram avaliadas e as eventuais mudanças que aconteceram em suas condições do trabalho.

Os dados foram elaborados com programa estatístico SPSS 15.0. Em primeiro lugar calculouse a taxa de resposta aos questionários, a seguir foram descritos (país a país) as características relativas a seus aspectos demográficos e de trabalho.

Considerado o número elevado de parâmetros empregados a fim estimar a percepção das condições de trabalho, foi aplicado a análise do conjunto “K-means Clustering”(21) para reduzir a uma classificação para grupos homogêneos de exposição "sugestiva”.

Com o teste $\chi^{2}$ ou o ANOVA (de acordo com a qualidade da escala), estimou-se o grau de associação entre os conjuntos baseados nas condições do trabalho percebidas e a coleta de informações objetivas sobre as características de trabalho em interface com o domicílio dos trabalhadores de enfermagem.

Outras variáveis foram: a instituição que o trabalhador de enfermagem trabalha; sua jornada de trabalho; avaliando em quais turnos pode-se atuar; número de turnos ao dia em que o profissional de enfermagem trabalha; número de turnos em um ano em que o trabalhador de enfermagem trabalha doente; os dias para capacitação profissional; o tempo para descansar; o número de crianças existentes no domicílio abaixo de sete anos e o tempo em minutos estimado para o deslocamento ao trabalho.

A fim de caracterizar a mudança nas condições de saúde e no bem estar da equipe de enfermagem entre os anos de 2003/2004 e 2002/ 2003 foi calculada a diferença entre as pontuações obtidas no seguimento e aquelas obtidas à época da primeira avaliação das variáveis: a exaustão emocional, saúde em geral, capacidade funcional no trabalho. Variáveis obtidas assim ( $\Delta$ da exaustão emocional, $\Delta$ da saúde em geral, $\Delta$ habilidade funcional no trabalho) foram empregadas a fim de verificar, por meio da análise de regressão linear, o valor preditivo dos conjuntos na mudança das normas de saúde e bem estar da equipe.

As análises de regressão linear foram comparadas entre o país, sexo e idade naqueles casos cuja associação com a variável de saúde e bem estar era resultado presente à análise invariável.

\section{RESULTADOS}

Em 2002-2003, excluído os dados de Finlândia, Grã Bretanha e Noruega, 30.607 enfermeiros (Tabela 2) responderam ao primeiro questionário com a taxa de 53.9\% (diferença entre os países: 43.2\% - 75.8\%); em 2003/2004, 20.582 enfermeiros responderam também ao segundo questionário com uma taxa de resposta total de 40.24\% (diferença entre os países: 25.1\% - 64.5\%). Já o resultado do presente estudo acerca de 19.099 enfermeiros, engloba as equipes que compilaram tanto no primeiro questionário quanto no segundo de seguimento, conforme mostra a Tabela 2.

Tabela 2 - Estudo NEXT - Questionário enviado para primeira avaliação em 2002/2003 e para a segunda avaliação (2003/2004)

\begin{tabular}{lcccccc}
\hline \multicolumn{1}{c}{ País } & \multicolumn{3}{c}{ Questionário 2002-2003 } & \multicolumn{3}{c}{ Questionário 2004-2005 } \\
\hline & enviados & recebidos & taxa de resposta & enviados & recebidos & taxa de resposta \\
Bélgica & 7.049 & 4.247 & $60,4 \%$ & 5.955 & 2.853 & $47,9 \%$ \\
Alemanha & 6.484 & 3.565 & $55,0 \%$ & 6.116 & 2.538 & $41,5 \%$ \\
França & 13.017 & 5.376 & $41,3 \%$ & 11.131 & 2.627 & $23,6 \%$ \\
Itália & 7.447 & 5.645 & $75,8 \%$ & 7.230 & 4.087 & $56,5 \%$ \\
Países Baixos & 9.309 & 4.024 & $43,2 \%$ & 9.675 & 2.433 & $25,1 \%$ \\
Polônia & 7.091 & 4.354 & $61,4 \%$ & 7.050 & 4.547 & $64,5 \%$ \\
Eslo váquia & 6.382 & 3.396 & $53,2 \%$ & 3.979 & 1.497 & $37,6 \%$ \\
Total de & 56.779 & 30.607 & $53,9 \%$ & 51.136 & 20.582 & $40,2 \%$ \\
questionários & & & & & & \\
\hline
\end{tabular}

Cogitare Enferm 2009 Jan/Mar; 14(1):11-21 
Quanto às características demográficas (sexo, idade, origem), situação familiar de vida (status, filhos e parentes carentes das curas) e do trabalho como o local da instituição, nível profissional, contrato, número de horas do trabalho, turnos, facilidade em encontrar outros locais de trabalho e intenção de sair do atual emprego foram observadas várias porcentagens entre os diferentes países (Tabela 3).

Tabela 3 - Estudo NEXT - Característica demográfica e laborativa de trabalhadores de enfermagem que responderam o questionário de base (2003-2004) e o de seguimento (2004-2005) nos diversos países europeus considerados neste estudo

\begin{tabular}{|c|c|c|c|c|c|c|c|}
\hline & $\begin{array}{l}\text { Bélgica } \\
\text { n. } 2971\end{array}$ & $\begin{array}{c}\text { Alemanha } \\
\text { n. } 2370\end{array}$ & $\begin{array}{l}\text { França } \\
\text { n. } 3459\end{array}$ & $\begin{array}{c}\text { Itália } \\
\text { n. } 3239\end{array}$ & $\begin{array}{c}\text { Países Baixos } \\
\text { n. } 2966\end{array}$ & $\begin{array}{l}\text { Polônia } \\
\text { n. } 2589\end{array}$ & $\begin{array}{c}\text { Eslováquia } \\
\text { n. } 1451\end{array}$ \\
\hline Sexo feminino & 91,2 & 81,6 & 87,6 & 71,3 & 89,3 & 98,7 & 97,11 \\
\hline Sexo masculino & 8,7 & 18,3 & 12,4 & 28,7 & 10,7 & 1,3 & 2,89 \\
\hline \multicolumn{8}{|l|}{ Faixa de idade } \\
\hline$<30$ anos & 26,5 & 23,1 & 24,0 & 16,1 & 25,1 & 16,7 & 22,5 \\
\hline 30-39 anos & 34,9 & 35,5 & 31,6 & 53,1 & 32,6 & 45,8 & 37,2 \\
\hline 40-49 anos & 30,0 & 31,8 & 31,2 & 24,3 & 31,2 & 32,5 & 31,7 \\
\hline $50-59$ anos & 8,4 & 9,2 & 12,9 & 6,3 & 10,9 & 5,0 & 8,6 \\
\hline$>59$ anos & 0,2 & 0,5 & 0,4 & 0,2 & 0,1 & 0,1 & 0,1 \\
\hline \multicolumn{8}{|l|}{ Lugar de nascimento } \\
\hline $\begin{array}{l}\text { Mesma região na qual } \\
\text { trabalha }\end{array}$ & 62,6 & 40,7 & 54,5 & 76,6 & 57,1 & 71,5 & 77,7 \\
\hline $\begin{array}{l}\text { Região diferente da qual } \\
\text { trabalha }\end{array}$ & 31,0 & 49,9 & 39,6 & 18,7 & 37,4 & 28,0 & 19,6 \\
\hline Outro país & 6,5 & 9,4 & 5,9 & 4,7 & 5,5 & 0,4 & 2,7 \\
\hline \multicolumn{8}{|l|}{ Sistema familiar } \\
\hline Solteiro & 11,1 & 21,8 & 15,1 & 12,5 & 12,3 & 4,7 & 5,7 \\
\hline Solteiro com criança & 5,4 & 6,5 & 8,4 & 3,9 & 2,7 & 5,7 & 7,4 \\
\hline Dois adultos & 24,2 & 31,3 & 23,8 & 27,3 & 31,8 & 18,1 & 19,2 \\
\hline $\begin{array}{l}\text { Ao menos dois adultos com } \\
\text { criança }\end{array}$ & 59,4 & 40,4 & 52,7 & 56,2 & 53,3 & 71,5 & 67,7 \\
\hline \multicolumn{8}{|l|}{$\begin{array}{l}\text { Tranquilidade para as } \\
\text { crianças quando trabalha }\end{array}$} \\
\hline Não & 19,0 & 19,3 & 20,9 & 29,4 & 7,7 & 17,7 & 15,0 \\
\hline A maior parte do tempo & 53,0 & 42,5 & 41,5 & 44,6 & 47,4 & 51,5 & 29,1 \\
\hline Sim quase sempre & 28,0 & 38,2 & 37,7 & 26,0 & 45,0 & 30,7 & 56,0 \\
\hline \multicolumn{8}{|l|}{$\begin{array}{l}\text { Atividade de assistência } \\
\text { a parentes necessitados }\end{array}$} \\
\hline Não & 91,8 & 92,7 & 93,7 & 86,4 & 91,4 & 76,5 & 83,0 \\
\hline Sim & 8,2 & 7,3 & 6,3 & 13,6 & 8,6 & 23,5 & 17,0 \\
\hline
\end{tabular}


Continuação

\begin{tabular}{|c|c|c|c|c|c|c|c|c|c|c|c|c|c|c|}
\hline \multicolumn{15}{|c|}{ Tipo de instituição } \\
\hline \multicolumn{2}{|l|}{ Hospital } & 54 & 1,9 & 80,2 & & 86,4 & & 91,4 & & 71,3 & & 86,9 & & 35,6 \\
\hline \multicolumn{2}{|l|}{ Asilo } & 5 & ,9 & 12,8 & & 12,4 & & 3,0 & & 18,3 & & 4,6 & & 12,6 \\
\hline \multicolumn{2}{|c|}{ Assistência domiciliar } & 39 &, 2 & 7,1 & & 1,2 & & 5,6 & & 10,4 & & 3,9 & & 1,9 \\
\hline \multicolumn{2}{|l|}{ Ambulatório } & & - & - & & - & & - & & - & & 4,6 & & - \\
\hline \multicolumn{15}{|c|}{ Nível ocupacional } \\
\hline \multicolumn{2}{|l|}{ (staff) } & 9 & 2 & 82,1 & & 95,2 & & 94,7 & & 96,9 & & 90,7 & & 39,5 \\
\hline \multicolumn{2}{|l|}{ Dirigente } & 5 & ,1 & 8,1 & & 0,6 & & 0,4 & & 1,7 & & 6,2 & & 5,2 \\
\hline \multicolumn{2}{|c|}{$\begin{array}{l}\text { Função de controle } \\
\text { provisória }\end{array}$} & 2 & 9 & 9,8 & & 4,3 & & 5,0 & & 1,4 & & 3,1 & & 5,4 \\
\hline \multicolumn{15}{|l|}{ Trabalho } \\
\hline \multicolumn{2}{|c|}{ De dia (horário regular) } & 18 & 3,8 & 9,4 & & 17,8 & & 10,4 & & 10,5 & & 3,5 & & 13,3 \\
\hline \multicolumn{2}{|c|}{ De dia (horário irregular) } & 8 & ,9 & 7,4 & & 4,0 & & 2,5 & & 7,2 & & 0,9 & & 1,9 \\
\hline \multicolumn{2}{|c|}{$\begin{array}{l}\text { Somente no turno da } \\
\text { noite }\end{array}$} & 4 & 8 & 6,4 & & 14,9 & & 0,1 & & 1,9 & & 0,0 & & 0,0 \\
\hline \multicolumn{2}{|c|}{$\begin{array}{l}\text { Com alternância de } \\
\text { turnos sem noite }\end{array}$} & 36 & 6,8 & 26,1 & & 45,4 & & 20,8 & & 19,0 & & 19,8 & & 15,9 \\
\hline \multicolumn{2}{|c|}{$\begin{array}{l}\text { Com alternância de } \\
\text { turnos com noite }\end{array}$} & 30 &, 7 & 50,7 & & 17,8 & & 66,3 & & 61,4 & & 75,8 & & 8,8 \\
\hline \multicolumn{15}{|l|}{ Tipo de contrato } \\
\hline \multicolumn{2}{|c|}{ Tempo indeterminado } & \multicolumn{2}{|c|}{94,3} & \multicolumn{2}{|c|}{91,1} & 95,2 & & 97,6 & & 94,5 & & 91,4 & & 4,5 \\
\hline \multirow[t]{5}{*}{ Tempo definido } & & 5 &, 7 & 8,9 & & 4,8 & & 2,4 & & 5,5 & & 8,6 & & 25,5 \\
\hline & & & & & & & & & & & & 10,6 & & 2,2 \\
\hline & \multicolumn{2}{|c|}{ Bélgica } & \multicolumn{2}{|c|}{ Alemanha } & \multicolumn{2}{|c|}{ França } & \multicolumn{2}{|c|}{ Itália } & \multicolumn{2}{|c|}{$\begin{array}{l}\text { Países } \\
\text { Baixos }\end{array}$} & \multicolumn{2}{|c|}{ Polônia } & \multicolumn{2}{|c|}{ Eslováquia } \\
\hline & \multicolumn{2}{|c|}{ n. 2971} & \multicolumn{2}{|c|}{ n. 2370} & n. 34 & & n. 32 & 239 & n. 29 & 966 & n. 2 & 589 & n. 1 & 451 \\
\hline & Média & DS & Média & DS & Média & DS & Média & DS & Média & DS & Média & DS & Média & DS \\
\hline $\begin{array}{l}\text { Criança com > } 7 \\
\text { anos }\end{array}$ & 0,4 & 0,7 & 0,2 & 0,5 & 0,4 & 0,7 & 0,4 & 0,7 & 0,4 & 0,8 & 0,3 & 0,6 & 0,2 & 0,5 \\
\hline $\begin{array}{l}\text { Facilidade de } \\
\text { novo posto de } \\
\text { trabalho }\end{array}$ & 4,0 & 0,9 & 3,2 & 1,1 & 3,4 & 1,2 & 4,0 & 1,4 & 4,0 & 0,7 & 1,5 & 0,8 & 1,6 & 0,9 \\
\hline $\begin{array}{l}\text { Horário de } \\
\text { trabalho }\end{array}$ & 30,8 & 7,6 & 31,6 & 9,9 & 33,9 & 6,2 & 35,3 & 7,1 & 25,3 & 8,3 & 40,2 & 3,9 & 38,6 & 5,4 \\
\hline
\end{tabular}

Os grupos foram identificados com base nas seguintes condições de trabalho percebidas: possibilidade de realização, qualidade de liderança, plenitude e significado profissional das tarefas, dos relacionamentos inter profissionais, suporte social, autonomia no trabalho, insegurança profissional, conflito casa-trabalho, acesso à organização, acesso à profissão, carga de trabalho, desequilíbrio entre o empenho e o reconhecimento no trabalho, incapacidade de se desligar das preocupações do trabalho. Os conjuntos obtidos distinguiram os trabalhadores de enfermagem em 5 grupos: um que considera todas as condições como “ótimas” (Grupo I), 
outro da "baixa participação de trabalho" (Grupo II), o terceiro com relação ao "baixo suporte social” (Grupo III), um quarto “da carga de trabalho excessiva” (Grupo IV) e no último que considera todas as condições como “péssimas” (Grupo V).

Na tabela 4 observa-se porcentagens dos trabalhadores de enfermagem por grupos, nos vários países.

Tabela 4 - Percentual de trabalhadores de enfermagem presentes em grupos, com base nos países considerados.

\begin{tabular}{lccccc}
\hline Grupos: & I & II & III & IV & V \\
& Ótimas condições & Baixa participação & Baixo suporte & Alta decadência & Péssimas condições \\
\hline Bélgica (n. 2984) & $30,7 \%$ & $17,1 \%$ & $12,0 \%$ & $31,0 \%$ & $9,2 \%$ \\
Alemanha (n. 2383) & $22,0 \%$ & $16,5 \%$ & $13,5 \%$ & $31,8 \%$ & $16,1 \%$ \\
França (n. 3469) & $25,5 \%$ & $13,7 \%$ & $30,2 \%$ & $20,8 \%$ & $9,8 \%$ \\
Itália (n. 3249) & $14,5 \%$ & $18,2 \%$ & $21,4 \%$ & $18,7 \%$ & $27,3 \%$ \\
Países Baixos (n. 2971) & $23,9 \%$ & $52,7 \%$ & $10,7 \%$ & $7,2 \%$ & $5,4 \%$ \\
Polônia (n. 2592) & $18,0 \%$ & $21,6 \%$ & $23,2 \%$ & $15,8 \%$ & $21,5 \%$ \\
Eslováquia ( n. 1451) & $24,8 \%$ & $17,4 \%$ & $15,0 \%$ & $30,3 \%$ & $12,5 \%$ \\
\hline
\end{tabular}

Sobre as variáveis idade e sexo, Bélgica, Alemanha e Eslováquia são caracterizadas na maior parte por uma carga de trabalho pesada, França e Polônia por escasso suporte social, Países Baixos por baixa participação no trabalho e na Itália por condições completamente negativas no trabalho. A Bélgica, embora haja uma porcentagem elevada de sujeitos com carga de trabalho elevado, é o país com a porcentagem mais elevada dos sujeitos (30\%) com "condições ótimas de trabalho".

A Tabela 5 apresenta resultados da associação das condições de vida objetivas e de trabalho. O grupo de trabalhadores da variável "circunstâncias ótimas", possuem menos crianças pequenas das quais devem tomar conta, perdem menos tempo para ir e retornar do trabalho faz pouco esforço físico no trabalho. A maioria destes trabalhadores de enfermagem desenvolve assistência domiciliar, podem descansar mais nos finais de semana e realizam um número inferior de turnos noturnos.

O grupo de trabalhadores da variável "pouco envolvido no trabalho" tem menos horas de trabalho, menos dias dedicados à formação e mais filhos abaixo de sete anos em domicílio; 35\% trabalham em ambulatórios, mais do que um quarto é comprometido com um turno somente do tipo noturno; o grupo de trabalhadores que relatou "elevada carga de trabalho" tem mais atividade relacionada ao esforço físico mobilizando no leito os pacientes. Para o grupo da variável "circunstâncias péssimas" tem todas as condições decadentes, sem perspectiva de melhora.

Tabela 5 - Características demográficas e laborativas “objetivas” dos trabalhadores de enfermagem incluídos nos diversos grupos

\begin{tabular}{lcccccc}
\hline & $\begin{array}{c}\text { Condições } \\
\text { ótimas } \\
\text { Média } \pm \text { DS }\end{array}$ & $\begin{array}{c}\text { Baixa } \\
\text { participação } \\
\text { Média } \pm \text { DS }\end{array}$ & $\begin{array}{c}\text { Baixo } \\
\text { Suporte } \\
\text { Média } \pm \text { DS }\end{array}$ & $\begin{array}{c}\text { Alta } \\
\text { decadéncia }\end{array}$ & $\begin{array}{c}\text { Péssimas } \\
\text { condições DS }\end{array}$ & $\begin{array}{c}\mathrm{p} \\
\text { Média } \pm \text { DS }\end{array}$ \\
\hline Trabalha doente (n. de vezes) & $2,5 \pm 7,4$ & $2,5 \pm 7,0$ & $4,1 \pm 11,0$ & $4,0 \pm 9,7$ & $5,1 \pm 12,6$ & $<.0001$ \\
Crianças em casa (n.) & $1,3 \pm 1,1$ & $1,5 \pm 1,1$ & $1,2 \pm 1,1$ & $1,2 \pm 1,1$ & $1,2 \pm 1,1$ & $<.0001$ \\
Crianças abaixo de 7 anos (n.) & $0,5 \pm 0,7$ & $0,6 \pm 0,8$ & $0,5 \pm 0,7$ & $0,5 \pm 0,7$ & $0,6 \pm 0,7$ & $<.0001$ \\
Distância casa-trabalho (minutos ida e & $39,5 \pm 32,6$ & $41,8 \pm 31,5$ & $45,3 \pm 36,7$ & $43,2 \pm 35,5$ & $46,8 \pm 38,0$ & $<.0001$ \\
volta) & & & & & & \\
Abaixar e encurvar-se escala ( 0-100) & $22,9 \pm 19,4$ & $23,0 \pm 19,4$ & $26,9 \pm 21,4$ & $28,6 \pm 21,6$ & $28,4 \pm 21,8$ & $<.0001$ \\
Horas de trabalho semanais & $32,8 \pm 8,2$ & $31,3 \pm 9,0$ & $34,8 \pm 8,1$ & $33,9 \pm 8,4$ & $35,2 \pm 8,2$ & $<.0001$ \\
\hline
\end{tabular}


Continuação

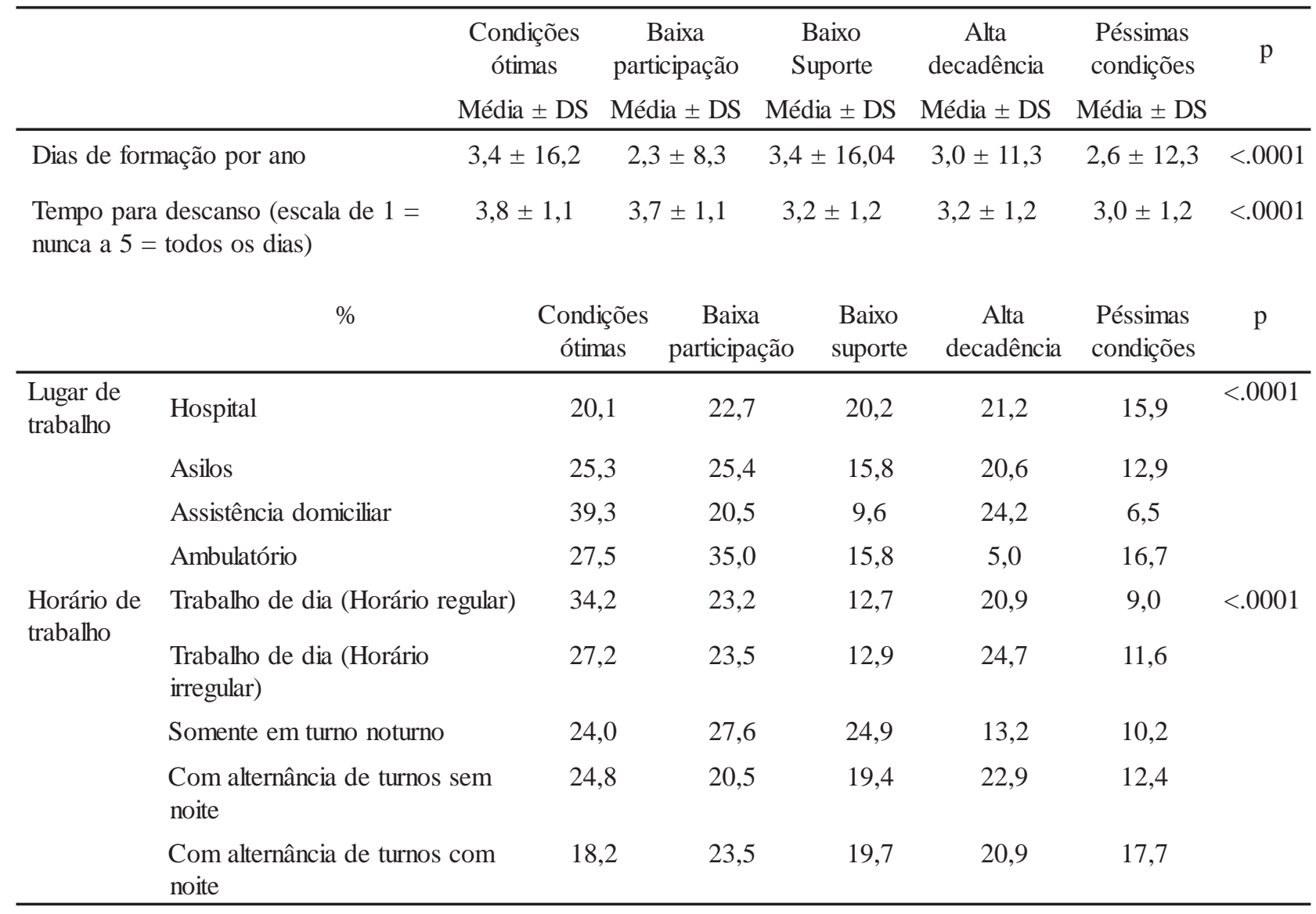

Calculadas as diferenças do estado de saúde e de bem estar no momento dos dois exames verificamos que estas diferenças variam de maneira significativa (ANOVA: p. < 0.05) de acordo com o sexo, idade, país e pertencentes ao conjunto determinado especificamente, como apresentado na Tabela 6. A exaustão é pior entre mulheres, em todas as faixas etárias, sobressaltando-se nos jovens na França, na Bélgica e na Eslováquia. Permanece inalterada nos outros países, na Polônia a exaustão é reduzida. A saúde em geral é pior em todos os países e em todos os grupos; a capacidade funcional é invariável na Itália e nos Países Baixos, eé pior nos outros países e em todo o conjunto a exceção daqueles definidos como “péssimas condições”.

Tabela 6 - Diferença entre os resultados obtidos nas escalas da saúde e bem estar entre as duas coletas (2003/ 2004 - 2002/2003) comparação por sexo, idade, país e grupos identificados

\begin{tabular}{|c|c|c|c|c|c|c|c|c|c|c|}
\hline \multirow{2}{*}{$\overline{\text { Sexo }}$} & & \multirow{2}{*}{$\frac{\Delta}{\mathrm{n} .}$} & \multicolumn{2}{|c|}{ Exaustão emocional } & \multirow{2}{*}{$\frac{\Delta}{\mathrm{n} .}$} & \multicolumn{2}{|c|}{ Saúde geral } & \multirow{2}{*}{$\frac{\Delta}{\mathrm{n} .}$} & \multicolumn{2}{|c|}{ Capacidade funcional } \\
\hline & & & Média & DS & & Média & DS & & Média & DS \\
\hline & Femino & 6261 & $-0,1$ & 0,9 & 6218 & $-2,9$ & 16,4 & 5431 & $-0,3$ & 5,0 \\
\hline & Masculino & 951 & 0,0 & 0,7 & 957 & $-3,3$ & 16,0 & 742 & $-0,4$ & 4,5 \\
\hline & p. & & $<.01$ & & & & & & & \\
\hline \multirow[t]{5}{*}{ Idade } & $<30$ & 1424 & $-0,2$ & 0,8 & 1428 & $-2,1$ & 17,2 & 1222 & $-0,5$ & 4,8 \\
\hline & $30-39$ & 2991 & $-0,1$ & 0,9 & 2975 & $-3,0$ & 16,3 & 2514 & $-0,2$ & 4,9 \\
\hline & $40-49$ & 2205 & $-0,1$ & 0,9 & 2186 & $-3,3$ & 16,2 & 1907 & $-0,3$ & 5,0 \\
\hline & $>50$ & 572 & $-0,1$ & 0,8 & 566 & $-3,2$ & 15,1 & 493 & $-0,4$ & 5,3 \\
\hline & & & $\mathrm{p}<.001$ & & & & & & & \\
\hline
\end{tabular}


Continuação

\begin{tabular}{|c|c|c|c|c|c|c|c|c|c|c|}
\hline \multirow[b]{2}{*}{ País } & \multirow[b]{2}{*}{$\mathrm{BE}$} & \multicolumn{4}{|c|}{ Exaustão emocional } & \multicolumn{2}{|c|}{ Saúde geral } & \multicolumn{3}{|c|}{ Capacidade funcional } \\
\hline & & 1114 & $-0,3$ & 0,7 & 1115 & $-2,7$ & 15,0 & 1043 & $-0,5$ & 4,6 \\
\hline & $\mathrm{DE}$ & 1104 & 0,0 & 0,7 & 1093 & $-1,3$ & 16,5 & 1032 & $-0,5$ & 4,9 \\
\hline & FR & 857 & $-0,6$ & 0,7 & 858 & $-2,0$ & 16,3 & 694 & $-0,4$ & 5,1 \\
\hline & IT & 1916 & 0,0 & 0,9 & 1905 & $-3,5$ & 16,1 & 1237 & 0,0 & 4,4 \\
\hline & NL & 876 & 0,0 & 0,5 & 872 & $-2,4$ & 14,4 & 862 & 0,0 & 4,5 \\
\hline & POL & 1072 & 0,2 & 1,1 & 1061 & $-4,7$ & 19,1 & 1043 & $-0,6$ & 6,2 \\
\hline & SLK & 277 & $-0,1$ & 0,8 & 275 & $-4,0$ & 16,7 & 264 & $-0,3$ & 4,0 \\
\hline & & & $\mathrm{p}<.001$ & & & p. $<001$ & & & $\mathrm{p}<.05$ & \\
\hline \multirow[t]{6}{*}{ Grupos } & $\begin{array}{l}\text { Péssimas } \\
\text { condições }\end{array}$ & 1120 & $-0,1$ & 1,0 & 1115 & $-1,6$ & 14,8 & 906 & 0,5 & 5,4 \\
\hline & $\begin{array}{l}\text { Alta } \\
\text { decadência }\end{array}$ & 1595 & 0,0 & 0,9 & 1579 & $-2,9$ & 16,6 & 1376 & $-0,2$ & 5,0 \\
\hline & Baixo suporte & 1288 & $-0,1$ & 0,9 & 1277 & $-2,7$ & 16,9 & 1061 & $-0,3$ & 5,2 \\
\hline & $\begin{array}{l}\text { Baixa } \\
\text { participação }\end{array}$ & 1120 & 0,0 & 0,7 & 1596 & $-2,7$ & 14,8 & 1409 & $-0,4$ & 4,6 \\
\hline & $\begin{array}{l}\text { Òtimas } \\
\text { condições }\end{array}$ & 1619 & 0,0 & 0,8 & 1612 & $-4,2$ & 16,1 & 1423 & $-0,9$ & 4,8 \\
\hline & & & $\mathrm{p}<.001$ & & & $\mathrm{P}<.01$ & & & $\mathrm{p}<.001$ & \\
\hline
\end{tabular}

A análise de regressão linear apresentada na Tabela 7, nas variáveis sexo, idade e país, confirmam uma redução significativa nos níveis de exaustão emocional na equipe de trabalhadores de enfermagem aferidos aos conjuntos “circunstâncias ótimas" e "baixo envolvimento".

Tabela 7: Análise de regressão linear estimada relacionada à condições de trabalho e saúde em um ano

\begin{tabular}{llll}
\hline & $\begin{array}{c}\Delta \text { Burnout: exaustão } \\
\text { emocional }\end{array}$ & $\Delta$ Saúde geral & $\Delta$ Capacidade funcional \\
\hline Grupo de referência: péssimas condições) & Coeficiente (IC: $95 \%)$ & Coeficiente (IC: 95\%) & Coeficiente (IC: $95 \%)$ \\
Alta decadência & $-0.0(-0.0,0.0)$ & $0.0(0.0,0.1)$ & $0.1(0.0,0.1)$ \\
Baixo suporte & $-0.0(-0.0,0.0)$ & $0.0(0.0,0.1)$ & $0.1(0.0,0.1)$ \\
Baixa participação & $-0.1(-0.1,-0,0)$ & $0.0(0.0,0.1)$ & $0.1(0.0,0.1)$ \\
Òtimas condições & $-0.1(-0.1,0.1)$ & $0.1(0.0,0.1)$ & $0.1(0.1,01)$ \\
\hline
\end{tabular}

IC 95\% = Intervalo de confiança de 95\%.

\section{ANÁLISE E CONSIDERAÇÕES FINAIS}

Estes resultados demonstram que a saúde e o bem estar dos trabalhadores de enfermagem dependem das condições do trabalho. Em relação a outros estudos das condições do trabalho e do bem estar dos trabalhadores de enfermagem este se baseia em um número elevado de trabalhadores de enfermagem, com um desenho longitudinal e, sobretudo, relaciona as condições, ao invés de analisá-las como se fossem isoladas ou independentes entre si. Relacionando as precedentes condições de saúde, foi possível verificar, após somente um ano de trabalho, um agravamento naqueles que se sentiram expostos às condições negativas, e uma melhoria naqueles que se sentiram expostos às condições ótimas ou aos que se envolvem 
em menor intensidade no processo de trabalho.

Os dados relacionados à percepção subjetiva das condições do trabalho são mais sensíveis do que as objetivas na previsão seja do dano e/ou da doença porque estas últimas dependem das vulnerabilidades individuais e habilidades de compreender e saber para enfrentar as situações ${ }^{(22)}$, entretanto, verificamos uma congruência discreta entre condições objetivamente graves e a percepção subjetiva entre os trabalhadores de enfermagem entrevistados.

As diferenças observadas entre os vários países da Europa refletem suas diversidades culturais, sócioeconômicas, organizacionais e da composição demográfica. A Itália, por exemplo, neste estudo, tem a porcentagem mais elevada em relação às "condições desfavoráveis ao trabalho” é também o país, entre aqueles listados, com a população mais baixa de trabalhadores de enfermagem por 100.000 habitantes (476, escala européia 476-2171) ${ }^{(23)}$ e, de acordo com os dados OECD $^{(24)}$, constatou-se baixo investimento de recursos destinados à saúde aos trabalhadores de enfermagem (8.5\% do produto interno bruto, escala européia 5.7-10.9); além disso, neste país, as razões políticas e econômicas impediram parcialmente uma gestão mais profissional e centrada no trabalhador de enfermagem ${ }^{(25)}$.

Embora na Europa haja uma harmonização das leis, existe ainda uma realidade complexa no que diz respeito aos trabalhadores de enfermagem. Os dados induzem a uma situação única: a quantidade reduzida de trabalhadores de enfermagem em instituições hospitalares, asilares e na assistência ambulatorial foi constatada. As dificuldades de conciliação entre trabalho e casa e a sustentação de jovens no processo de trabalho foram constatadas.

A fim de planejar as intervenções que podem reduzir o turnover e a deficiência da equipe de trabalhadores de enfermagem na Europa, é importante que se considere o processo de trabalho existente e os mecanismos para a redução da exposição destes trabalhadores; é importante também compreender que a saúde e o bem estar não podem prescindir das circunstâncias reais, porque são estas situações que permitem aos trabalhadores desenvolver suas atividades laborativas até a aposentadoria, situação que pode reduzir o abandono precoce no trabalho, bem como reduzir a carência significativa destes trabalhadores existente nesta área.

Na definição da variável "promoção da saúde”, estão compreendidos fatores organizacionais e psicossociais que se associaram ao conceito da saúde e do bem estar como clima de trabalho socialmente suportável, autonomia, satisfação, horários flexíveis de trabalho (na exigência do trabalhador) e a participação na programação, organização do trabalho e do ambiente físico ${ }^{(6,8)}$. Estes programas, também assegurariam o desenvolvimento de comportamentos adequados e saudáveis no trabalho. Na literatura já há alguns bons exemplos deste tipo de participação na saúde, como um exemplo aqueles de Bringsen na Suécia e em Erlingsdóttir H, na Islândia ${ }^{(26)}$.

\section{REFERÊNCIAS}

1. Simoens S, Villeneuve M, Hurst J. Tackling nurse shortages in OECD countries. OECD health working papers. OECD Health Working Papers: DELSA/ELSA/ WD/HEA 1; 2005.

2. Hasselhorn HM, Tackenberg P, Muller BH. Working conditions and intent to leave the profession among nursing staff in Europe. SALTSA - Joint Programme for Working Life Research in Europe. Report N 7; 2003.

3. Hasselhorn HM, Müller BH, Tackenberg P, NEXT Scientific Report. University of Wuppertal; July 2005.

4. Camerino D, Conway PM, Van der Heijden BIJM, EstrynBehar M, Costa G, Hasselhorn HM. Age-dependent relationships between work ability, thinking of quitting the job, and actual leaving among Italian nurses: A longitudinal study. Int J Nurs Stud. 2008; 45(1):164559.

5. Tai TW, Bame SI, Robinson CD. Review of nursing turnover research, 1977-1996. Soc Sci Med. 1998; 47(12):1905-24.

6. Laureen JH, O’Brien-Pallas L, Duffield C, Shamian J, Buchan J, Hughes F et al. Nurse turnover: A literature review. Int J Nurs Stud. 2006;43(2):237-63.

7. Coomber B, Barriball KL. Impact of job satisfaction components on intent to leave and turnover for hospital-based nurses: A review of the research literature International Int J Nurs Stud. 2007;44(2):297-314.

8. O’Brien-Pallas L, Griffin P, Shamian J, Buchan J, Duffield $\mathrm{C}$, Hughes $\mathrm{F}$ et al. The Impact of nurse turnover on patient, nurse, and system outcomes: a pilot study and focus for a multicenter international study. Policy, Politics, \& Nursing Practice 2006;7(3):169-79.

9. Estryn-Béhar M, Le Nézet O, Van der Heijden BIJM, Ogiñska H., Camerino D., Conway PM, et al.. Inadequate 
teamwork and burnout as predictors of intent to leave nursing according to seniority. Stability of associations in a one-year interval in the European NEXT Study. Ergonomia 2007;29(3-4):225-33.

10. McVicar A. Workplace stress in nursing: a literature review. J Adv Nurs. 2003; 44(6): 633-42.

11. Benner P, Sheets V, Uris P, Malloch K, Schwed K, Jamison D. Individual, practice, and system causes of errors in nursing: a taxonomy. J Nurs Admin. 2002;32(10):509-23.

12. Camerino D, Conway PM, Estryn-Béhar M. Fear of making errors in young and old Italian nurses. Ergonomia 2007, 29(3-4):211-17.

13. Gerberich, SG, Church TR, McGovern PM, Hansen HE, Nachreiner NM, Geisser MS et al.. An epidemiological study of the magnitude and consequences of work related violence: the Minnesota Nurses' Study. Occup Environ Med. 2004;61(6):495-503.

14. Camerino D, Estryn-Behar M, Conway PM, van Der Heijden BIJM, Hasselhorn HM. Work-related factors and violence among nursing staff in the European NEXT study: A longitudinal cohort study. Int J Nurs Stud. 2008; 45(1):35-50.

15. Kristensen TS. A new tool for assessing psychosocial factors at work: the Copenhagen Psychosocial Questionnaire. National Institute of Health: Copenhagen; 2000.

16. Netemeyer RG, McMurrian R, Boles JS. Development and validation of Work-Family Conflict and FamilyWork Conflict Scales. Journal of Applied Psychology. 1996;81(4):400-10.

17. Siegrist J. Adverse health effects of high effort - low reward conditions at work. Journal of Occupational Health Psychology. 1996; 1:27-43.

18. Tuomi K, Ilmarinen J, Jahkola A et al. Work ability. 2nd ed. Finnish Institute of Occupational Health: Helsinki; 1998.

19. Hasselhorn HM, Muller BH, Freude G, Tempel J, Kaluza S. The Work Ability Index (WAI) - establishment of a German WAI-network. In: Costa G, Goedhard JAW, Ilmarinen J (Eds.), Assessment and Promotion of Work Ability, Health and Well-being of Ageing Workers. Elsevier, International Congress Series 1280, Amsterdam 2005, p. 292-5.

20. Kristensen TS, Borritz M. Copenhagen burnout inventory: normative data from representative danish burnout. National Institute of Occupational Health: Copenhagen; 2001.

21. Romesburg HC. Cluster Analysis for Researchers. North Carolina (United State of America); Lulu press, 2004.

22. Cox T. Stress. MacMillan, London; 1978.

23. WHO. Global Atlas of infectious diseases. 04.26.2005 http://www.who.int/globalatlas/

24. OECD. OECD Health data, 3rd edition, 2004. Available at: http://www.oecd.org/document/16/ 0,2340,en_2825_495642_2085200_1_1_1_1,00.html

25. De Pietro C. Gestire il personale nelle aziende sanitarie italiane. Contesto, politiche e strumenti. Ed. Mc GrawHill: Milano; 2005.

26. Leino T, Olsson K. Workplace Health Promotion Practice evaluation. Finnish Institute of Occupational Health, Helsinki (Finland); 2004. 